\title{
Sobre el empleo de aditivos de molienda en la molturación de cemento (*)
}

\section{INTRODUCCION}

Los aditivos de molienda son sustancias sólidas, líquidas o gaseosas que se adicionan en pequeñas cantidades a los materiales a moler con objeto de aumentar el aprovechamiento de la energía durante la trituración. Hoy día se utilizan como aditivos para la molienda de cemento, principalmente líquidos orgánicos. Su primer empleo data de hace unos 40 años, pero en Alemania sólo en los últimos 15 años se ha tratado más intensamente su aprovechamiento para la industria. En 1968, aproximadamente un $10 \%$ del cemento producido en Alemania se molió con ayuda de aditivos.

\section{EVOLUCION}

\subsection{Patentes y solicitudes de patentes}

Una de las primeras referencias de empleo de aditivos de molienda en la industria de cemento se encuentra en una patente británica de 1930, (1), en la que se alude a la adición de resinas en pequeñas cantidades. El primer aditivo líquido - una mezcla de trietanolamina y sales de calcio solubles de ácidos ligninsulfónicos (marca comercial TDA) - se patenta en EE.UU. a partir de 1935 (2).

En Alemania, en 1952 se patentó un método para la molienda fina de cemento empleando pez, brea o aceites viejos, (3).

A partir de 1954 en la Gran Bretaña, (4), y de 1956 en Austria, (5), se patenta "la molienda en atmósfera gaseosa con ayuda de materiales que influyen en la superficie".

Además, en los años 1960 y 1962 se solicitó la patente para fenol, (6) y (7), y en 1965 para la solución acuosa de acetato de etanolamina acetilado (marca comercial HEA-2) como aditivo de molienda, (8) y (9). Este acetato de amina ha sustituido al TDA desarrollado por el mismo fabricante.

\subsection{Literatura}

En 1936, H. L. Kennedy, (10), informa sobre el aumento de rendimiento en los molinos de un 30 a un $50 \%$, adicionando TDA. En comparación con los cementos molidos sin

(*) Agradecemos al autor y a la revista Zement-Kalk und Gips, Bauverlag GmbH, Wiesbaden - Alemania, su autorización en la transcripción del presente artículo. 
TDA, se observaron mayores resistencias después del endurecimiento. Además, el cemento era menos delicado durante el almacenamiento. E. R. Dawley, (11), confirma estos resultados. En esta época también se realizaron ensayos con TDA, (12), en fábricas de cemento alemanas.

F. C. Bond y F. T. Agthe, (13), por adición de pequeñas cantidades de agua, soluciones de lignina y carbón, pudieron reducir el recubrimiento de los cuerpos molturadores y consiguieron mayores superficies específicas con el mismo tiempo de molienda. C. W. Sweitzer y A. E. Craig, (14), lograron una duración de molienda aproximadamente un $30 \%$ más pequeña con adiciones de 0,3 y 1,3 \% de carbón coloidal en molinos de laboratorio. En 1944, E. R. Dawley, (15), informó sobre mejoras en la molienda de cemento en molinos de laboratorio, con adiciones de resinas vinílicas, aceite de bacalao y grasa de vaca.

Según A. Masel, (16), en una fábrica de cemento rusa se consiguió un aumento en el producto molido entre un 10 y $15 \%$ por adición de un $0,075 \%$ de "Soapstock": un producto que se obtiene al refinar aceite de algodón. A. F. Taggart, (17), R. F. Blanks y L. H. Kennedy, (18), así como H. E. Rose y R. M. E. Sullivan, (19), destacan el efecto dispersante de los aditivos de molienda. Por esto se obtienen los mayores aumentos de producción con aditivos de molienda en aquellos molinos en que se produce una fuerte aglomeración del material fino y se recubren los elementos molturadores, (20).

Con frecuencia, en los ensayos de laboratorio ciertos materiales se ensayaron sólo por su composición química definida, con el fin de obtener indicaciones sobre los efectos en determinados grupos de materiales. J. Grzymek y otros, (21), utilizaron ácido oleico, ácido naftolsulfúrico y agua para sus amplios ensayos con clínkeres de diferente composición mineralógica. Redujeron el tiempo original de molienda hasta $1 / 7$ en un molino de bolas de $150 \times 140 \mathrm{~mm}$ con $200 \mathrm{~g}$ de material. Para los diferentes materiales, las cantidades favorables de aditivo dependían del tipo de clínker y del aditivo empleado. Las adiciones de agua, estearato sódico, alcohol etílico, acetona, ácido oleico, estearina y nafteno, lo mismo que las de carbón coloidal, favorecían el proceso de desmenuzamiento en un molino de varillas de laboratorio, (22). La acetona, el ácido oleico, el alcohol y la trietanolamina mejoraron el proceso de molienda en un molino vibratorio, (23). El agua solamente era favorable hasta un $1 \%$; por encima de esta cantidad se producía aglomeración. En los ensayos del CERILH, (24), en molinos de bolas de laboratorio se adicionaron, entre otras cosas, sustancias para retrasar la molienda (o sea, acelerar la aglomeración) y para formar poros de aire.

En las fábricas de cemento rusas, por lo visto, se emplea, con mucha frecuencia, la trietanolamina como aditivo de molienda, [(25), (26), (27), (28), (29) y (30)].

F. G. Mardulier, [(31), (32) y (33)], describe los efectos de los aditivos de molienda y su influencia en el comportamiento al flujo y al tiempo de permanencia del material en el molino. Hace notar que las condiciones mecánicas de la instalación de molienda tienen que adaptarse a las condiciones de molido alteradas por los aditivos. Sus informaciones se basan en experiencias hechas con HEA-2.

De los ensayos de E. Ziegler, [(34) y (35)], realizados en el Instituto de Investigación de la Industria del Cemento en Düsseldorf, inspirados por los trabajos de P. A. Rehbinder y otros, (36), se desprende que la resistencia a la conminución de cuerpos sólidos se reduce por absorción de moléculas polares activas en su superficie.

En otros ensayos, [(37), (38) y (39)], sobre todo con alcoholes más elevados, el etilenglicol resultaba un aditivo de molienda eficaz y barato y que no tenía gran influencia en las pro- 
piedades del cemento. Por esta razón, en 1.961, el Instituto realizó los primeros ensayos de fábrica con etilenglicol.

\section{ENSAYOS DE LABORATORIO REALIZADOS POR EL INSTITUTO DE INVESTI- GACION DE LA INDUSTRIA DEL CEMENTO EN DÚSSELDORF}

Los ensayos se realizaron en un molino calentable con un diámetro de $610 \mathrm{~mm}$ y una longitud de $610 \mathrm{~mm}$. Como material de molienda se empleó clínker de tamaño inferior a $5 \mathrm{~mm}$ o cemento premolido con una superficie específica, según Blaine, de 1.500 a 2.000 $\mathrm{cm}^{2} / \mathrm{g}$. En la cámara de molienda, adaptándose a las condiciones de servicio de los grandes molinos, podía conseguirse una temperatura de $100^{\circ}$ a $120^{\circ} \mathrm{C}$. Los aditivos de molienda se encontraban presentes desde el principio del ensayo.

Algunos de los resultados de estos ensayos están representados en la figura 1. Se encuentra que la superficie específica en presencia de aditivos de molienda aumenta más rápidamente que al moler sin aditivos.

Otros resultados de ensayo, (40), demuestran que el ahorro de trabajo en la trituración al emplear aditivos de molienda aumenta con la superficie específica del material a moler (véase figura 2). Este resultado coincide cualitativamente con los resultados de fábrica.

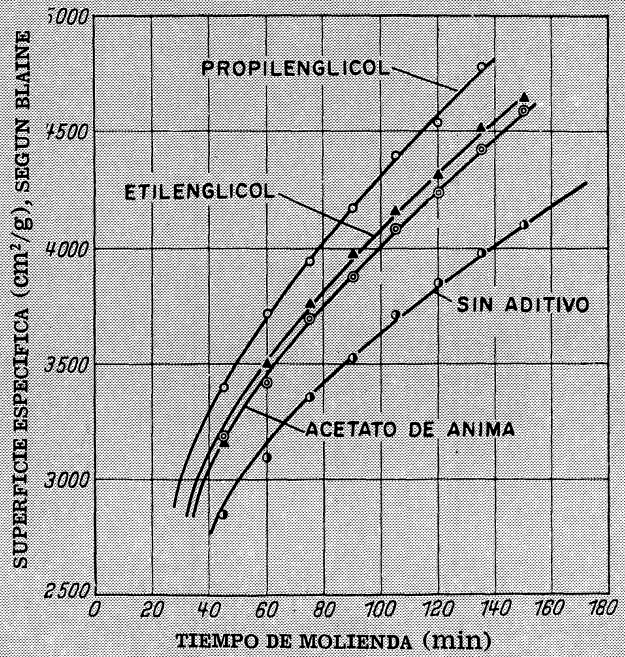

Fig. 1.-Superficie especifica en función del tilempo de mollenda, preparando cemento en un mollino de bolas de laboratorio.

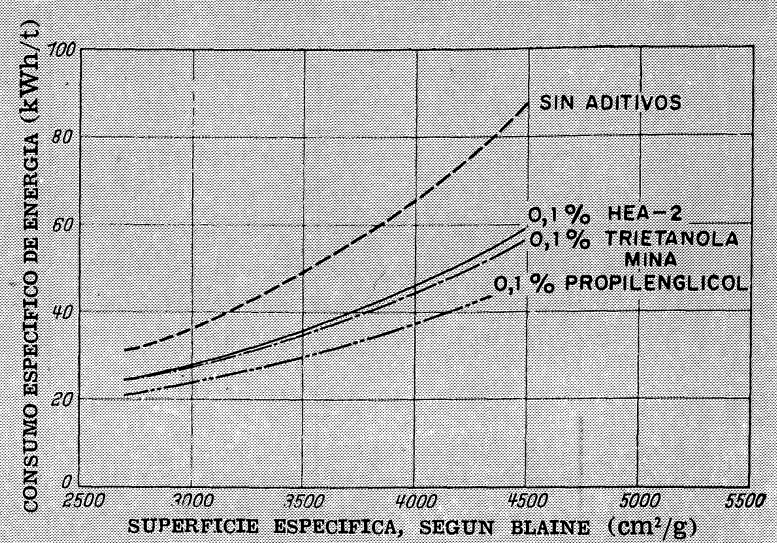

Fig. 2.-Consumo especifico de energía en función de la superficie especiflea mollendo cemento en molinos de ensayo.

En los molinos de laboratorio puede compararse el efecto de diferentes adiciones bajo las mismas condiciones. Pero, según la experiencia, los ensayos de laboratorio no son suflcientes para prever los efectos de los aditivos de molienda en molinos de fábrica. Sin embargo, estos ensayos son necesarios para que pueda estudiarse la influencia sobre el endurecimiento del cemento, antes de emplear un determinado aditivo de molienda en la fábrica.

\section{RESULTADOS DE FABRICA}

En los primeros ensayos de fábrica realizados por el Instituto de Investigación se estudió la influencia del etilenglicol sobre el rendimiento de un molino de refino de $1,2 \times 4,2 \mathrm{~m}$. 
El molino se alimentó con cemento que había sido premolido a una superficie específica de $2.800 \mathrm{~cm}^{2} / \mathrm{g}$. Como resultado de este ensayo, representado en la tabla 1 , puede decirse que el rendimiento del molino aumentó en un $100 \%$. Debido a los ensayos realizados en el pequeño molino industrial, algunas fábricas de cemento decidieron realizar ensayos a escala natural con aditivos de molienda. Primeramente se empleaba, exclusivamente, etilenglicol. Pero en los últimos años se ensayaron también otros productos químicos con efectos sobre la superficie, tanto en el laboratorio como en la fábrica. Entre ellos resultaron adecuados el propilenglicol, que hoy día ha suprimido en gran escala el etilenglicol, y la trietanolamina análoga al HEA-2, todavía no muy conocido en Alemania y que se usa sobre todo en EE.UU. [Contiene una parte importante de trietanolamina, (31)]. Los resultados de los ensayos de fábrica, ordenados según la superficie específica del cemento molido, figuran en la tabla 2. La mayoría de estos valores fue averiguada por las fábricas en servicio continuo. Los aumentos del rendimiento que figuran en la columna 6 de la tabla 2 son de 10 a $50 \%$. Los valores del aprovechamiento de energía representados en las columnas 7 y 8 hacen ver que las ventajas no solamente consisten en el aumento del rendimiento de las instalaciones.

T A B L A 1

Resultados de fábrica con etilenglicol en un molino de refino de $1,2 \times 4,2 \mathrm{~m}$

\begin{tabular}{|c|c|c|c|}
\hline Cantidad añadida & $\begin{array}{c}\text { Superficie específica } \\
\text { del producto } \\
\text { acabado }\end{array}$ & \multicolumn{2}{|c|}{ Producción } \\
\hline$(\%)$ & real & aumento \\
\hline- & $\left(\mathrm{cm}^{2} / \mathrm{g}\right)$ & $(\mathrm{t} / \mathrm{h})$ & $(\%)$ \\
\hline 0,009 & 3.770 & 2,0 & - \\
0,02 & 3.880 & 2,2 & 10 \\
0,03 & 3.850 & 2,4 & 20 \\
0,05 & 3.840 & 2,7 & 35 \\
0,1 & 3.770 & 3,0 & 50 \\
-3.780 & 3,9 & 95 \\
\hline 0,1 & 4.430 & 2,0 & - \\
- & 4.440 & 1,0 & 100 \\
\hline
\end{tabular}

Los resultados de otros ensayos de fábrica pueden verse en la figura 3. Está representado el aprovechamiento de energía en función de la superficie específica del cemento. Además, está marcado el margen de dispersión de los valores de molturabilidad de los respectivos clínkeres. En el campo del PZ 275 con superficies específicas de 2.500 a 3.000 $\mathrm{cm}^{2} / \mathrm{g}$ existen pocos valores, porque el aumento de rendimiento con esta finura, en general, no es suficientemente grande para compensar los costos del aditivo de molienda. En el campo de finura, de alrededor de $5.000 \mathrm{~cm}^{2} / \mathrm{g}$, solamente se disponía de un valor de comparación sin aditivo, porque con el PZ 475, antes de emplear aditivos de molienda líquidos, se empleaba, en general, carbón como coadyuvante en la molturación. 


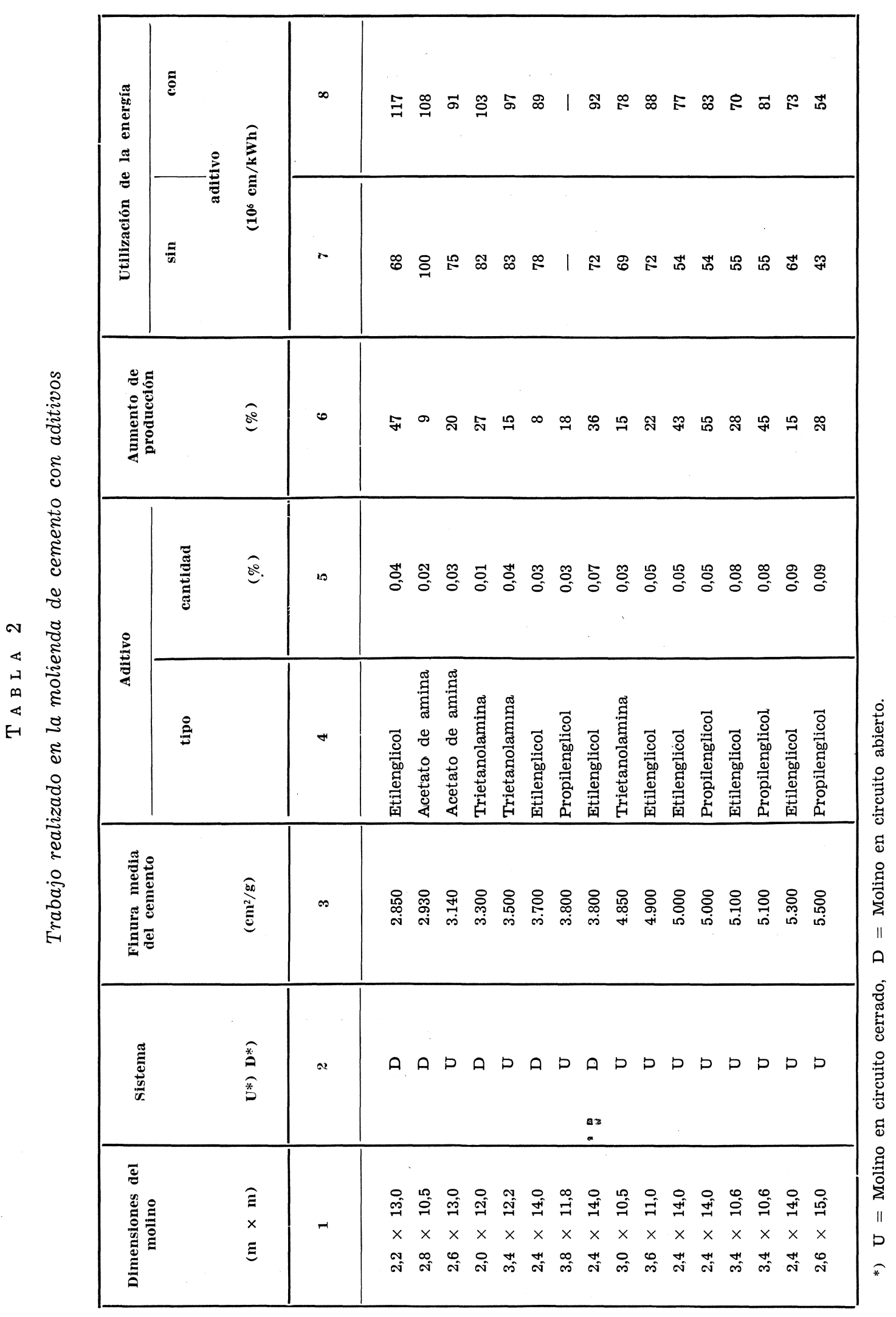

37 


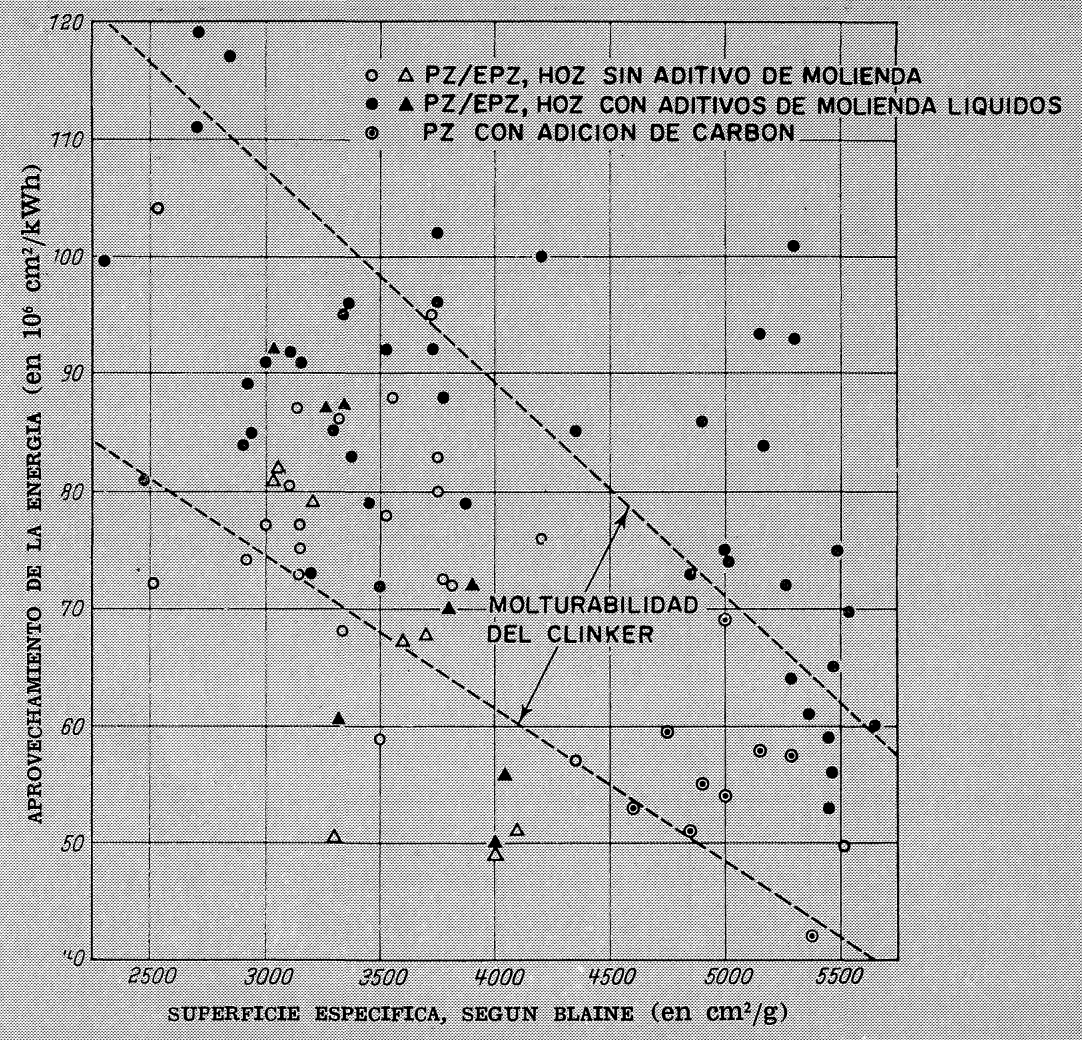

Fig. 3-Estimación del trabajo de la mollenda de cemento sin y con aditivos de molienda: aprovechamiento de la energía en función de la superfole especitica.

La tabla 3 muestra los valores medios del aumento del rendimiento en molinos de fábrica gracias a los aditivos de molienda, y están ordenados según el tipo resistente del cemento portland. Los valores indicados según la experiencia en fábricas alemanas coinciden con los valores conocidos por la literatura, [(25), (26), (27), (28) y (29)].

\section{T А в L A 3}

Aumento de la producción empleando aditivos en la molienda de cemento

\begin{tabular}{|c|c|c|c|}
\hline Cemento & $\begin{array}{c}\text { Superficie específica } \\
\left(\mathbf{c m}^{2} / \mathbf{g}\right)\end{array}$ & $\begin{array}{c}\text { Aumento de } \\
\text { producción } \\
(\%)\end{array}$ & $\begin{array}{c}\text { Cantidad añadida } \\
(\%)\end{array}$ \\
\hline PZ 275 & $2.400-3.000$ & bis 10 & $0,01-0,03$ \\
PZ 375 & $3.000-4.000$ & 10 bis 30 & $0,02-0,06$ \\
PZ 475 & $4.000-5.500$ & 25 bis 50 & $0,04-0,1$ \\
\hline
\end{tabular}

El aumento del rendimiento no solamente depende de la clase y cantidad del aditivo de molienda, sino también de las condiciones de servicio, tales como: la ventilación y tem- 
peratura en el molino, de la relación de las masas de cuerpos de molienda con el material a moler, del tamaño absoluto de los cuerpos molturadores y de la finura deseada del producto final, lo mismo que de las propiedades físicas y químico-mineralógicas del material que de molienda, de las cuales hasta ahora no se sabe mucho. La influencia del material que se muele se deduce, por ejemplo, del hecho de que en algunos clínkeres los glicoles son menos eficaces que la trietanolamina o viceversa. Las diferencias se confirmaron por ensayos en un molino de laboratorio y, probablemente, se deben al distinto comportamiento en la absorción de los clínkeres.

Contrariamente a los efectos, a veces considerables, de los aditivos al moler clínker de cemento portland, predominantemente cristalino, al moler cemento siderúrgico sólo se alcanzan mejoras reducidas, lo cual puede deducirse de la figura 3. En general, al aumentar la proporción de arena siderúrgica, predominantemente amorfa y vitrificada, la eficacia de los aditivos de molienda se reduce. Pero frente a la mayoría de los ensayos de fábrica en que no se producían ninguna o solamente leves mejoras en el rendimiento, se presentan algunos resultados de fábrica que corresponden con los del cemento portland. En un molino con circulación de $3,0 \times 12,5 \mathrm{~m}$, por ejemplo, al moler $\mathrm{HOZ}$ con un $70 \%$ de arena siderúrgica, a $3.700 \mathrm{~cm}^{2} / \mathrm{g}$ de superficie específica, con un $0,05 \%$ de etilenglicol, el rendimiento aumentó en un $20 \%$, y con un $60 \%$ de arena siderúrgica y $3.900 \mathrm{~cm}^{2} / \mathrm{g}$ aumentó incluso hasta un $25 \%$.

De los actuales valores de fábrica no puede deducirse si los aditivos de molienda son más eficaces en molinos en circuito abierto o con circulación. En los molinos en circuito abierto, el aprovechamiento de energía al aumentar la finura disminuye más que en los molinos con circulación. Eso se debe a que el molido fino en paso continuo se realiza en presencia de una mayor proporción de material fino. Puede producirse aglomeración, lo cual perturba el proceso de molienda. Este impedimento, en general, se hace notar en finuras de 3.000 a $5.000 \mathrm{~cm}^{2} / \mathrm{g}$. Pero ésta también es la zona en que los aditivos de molienda son especialmente eficaces. Por esta razón, en los molinos en circuito abierto que muelen por encima de $3.500 \mathrm{~cm}^{2} / \mathrm{g}$ pueden esperarse mayores aumentos del rendimiento con los aditivos de molienda que en los molinos con circulación. Así se explican los grandes aumentos del rendimiento del molino de refino de $1,2 \times 4,2 \mathrm{~m}$ que trabajaba en circuito abierto (tabla 1).

\section{SUMINISTRO, ALMACENAJE E INCORPORACION DE LOS ADITIVOS DE MOLIENDA}

Los aditivos de molienda líquidos se suministran en barriles o en cisternas. Con un consumo anual de 25 a $30 \mathrm{t}$, resulta ya más adecuado el suministro en cisternas. Dados los pequeños costos de adquisición y de transporte dentro de la fábrica de los aditivos de molienda, las instalaciones necesarias para el almacenaje se amortizan en 1 ó 2 años.

Para almacenar los aditivos de molienda suministrados en cisternas resultan indicados los silos de obra o depósitos de combustible corrientes con una capacidad de $10 \mathrm{~m}^{3}$ por lo menos.

En ensayos de corta duración, en general resulta suficiente añadir los aditivos de molienda directamente sobre el material a moler. Para el servicio continuo son indicadas bombas de dosificación que incorporan al material un flujo continuo de aditivo. Estas bombas de ruedas dentadas o de membrana deberían tener un margen de ajuste de por lo menos 1:50. Además, hay que tener en cuenta que los aditivos de molienda se distinguen por su viscosidad, lo cual se hace notar en la capacidad de la bomba. 
Los precios de las bombas de dosificación oscilan, según su capacidad, exactitud de dosificación, margen de regulación, etc., entre 500 y 3.000 DM.

Como estas bombas dosifican volumétricamente, hay que tener en cuenta la relación entre la temperatura, la viscosidad y la densidad de los aditivos de molienda para que la cantidad en $\mathrm{kg} / \mathrm{h}$ se mantenga constante durante el molido. Es aconsejable un dispositivo de calefacción en el depósito o en el conducto de alimentación. Resultan necesarios otros dispositivos de medición y mezcla cuando a los aditivos de molienda se adiciona una solución acuosa. La mezcla con agua puede realizarse mecánicamente, por aire a presión, o por medio de una bomba de circulación.

En la figura 4 se ve una instalación para el almacenamiento e incorporación de aditivos. El aditivo va a una bomba de presión y pasa por un calentador, un regulador del flujo y un contador hacia la entrada del molino donde con aire a compresión entra por una tobera. Es necesaria una válvula magnética acoplada con el accionamiento del molino o con el dispositivo de dosificación, la cual, en caso de fallar la alimentación de material, interrumpe la adición de aditivos. Del trabajo de N. C. Ludwig y L. M. O'Hara, (41), puede deducirse un ejemplo de los esquemas de conducciones y de flujo de una instalación de dosificación de aditivos de molienda con una regulación de cantidades proporcional.

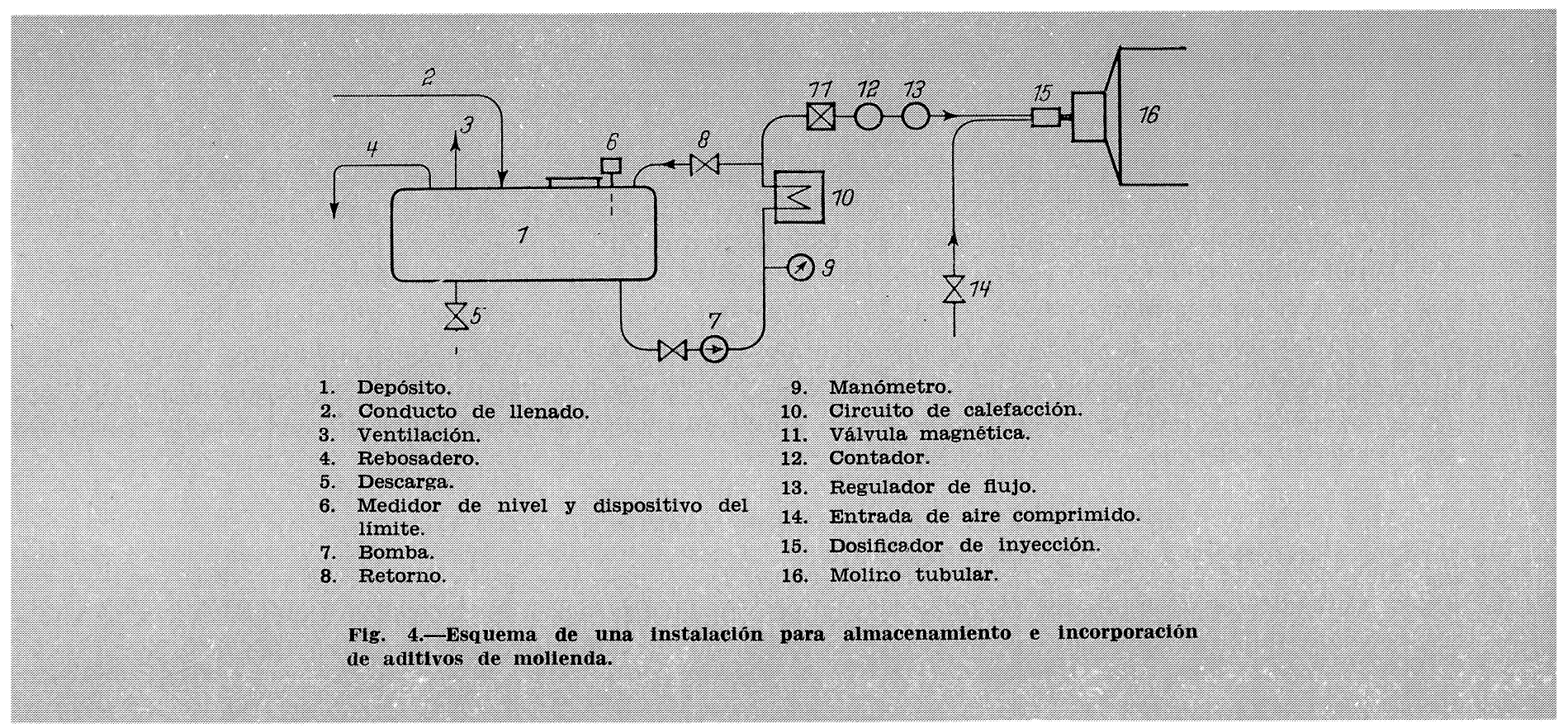

Los aditivos muy fluidos tales como el etilenglicol o el propilenglicol se utilizan, en general, sin diluir. En cambio, los líquidos con mayor viscosidad se transportan mejor en solución acuosa. Por la dilución con agua se trata de lograr una mejor distribución del aditivo en la superficie relativamente grande del material a moler fino, (32).

En el flujo por gravedad o trabajando con bomba de dosificación que solamente puede superar la resistencia del conducto de transporte entre la bomba y el molino, el aditivo de molienda se incorpora sobre el flujo de clínker o material granulado fuera del recinto. Pero el resultado óptimo sólo puede lograrse cuando los aditivos se ponen en íntimo contacto con el grano fino que se produce durante la trituración. Esto se consigue mejor pa- 
sando el aditivo por una tobera a la zona de molido y, mejor todavía, incorporándolo en la cámara de refino con bombas de dosificación que trabajen a varias atmósferas de presión o por pulverización con aire comprimido (fig. 5). Muy raras veces el fabricante suministra los molinos con estos dispositivos y, posteriormente, suele ser muy complicada la introducción en la cámara de refino. Al construir los molinos nuevos debería tenerse en cuenta la posibilidad de introducción de aditivos.

Los ensayos sobre aplicación de aditivos de molienda solamente se conocen de la literatura. S. M. Rojak y otros, (27), hablan de un molino de cemento que sin aditivo producía 22,4 t/h; 25,6 t/h, al echar aditivo sobre el clínker fuera del molino y 29,4 t/h, al introducirlo por toberas en la cámara de preparación. Los resultados de fábrica, de los

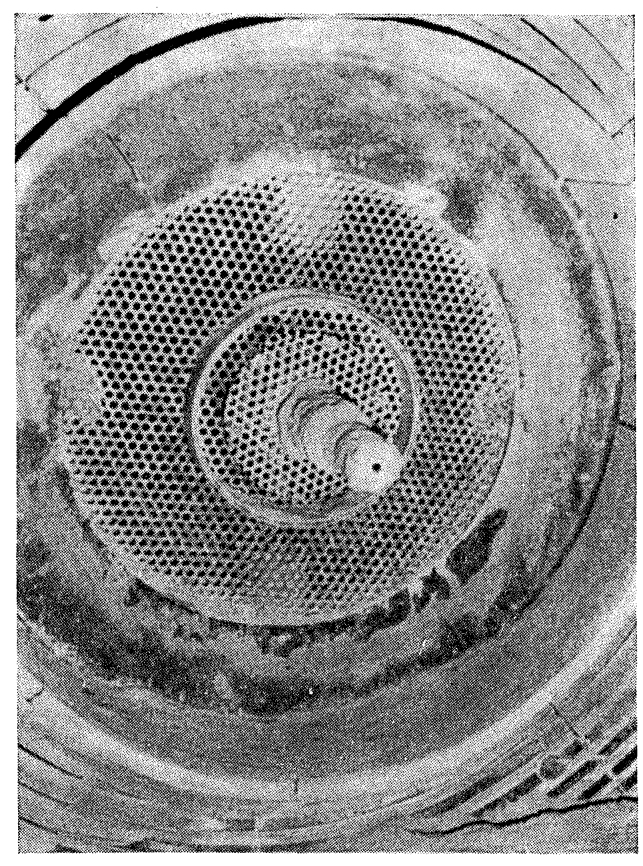

Fig. 5.-Dosificador de inyección a la salida de la cámara de refino.
T A B L A 4

Aumentos de la producción con aditivos [según F. J. Mardulier (32)]

\begin{tabular}{|l|c|c|}
\hline \multicolumn{1}{|c|}{ Lugar de trabajo } & $\begin{array}{c}\text { Cantidad } \\
\text { añadida } \\
(\%)\end{array}$ & $\begin{array}{c}\text { Aumento de } \\
\text { 1a producción } \\
(\%)\end{array}$ \\
\hline Cinta de clínker & 0,12 & 7 \\
Cámara I & 0,12 & 15 \\
\hline Transportador de & 0,15 & 0 \\
clínker & 0,13 & 0 \\
\hline Cinta de clínker & 0,12 & 0 \\
Cámara I & 0,12 & 14 \\
\hline Cinta de clínker & 0,12 & 17 \\
Cámara I & 0,12 & 22 \\
\hline Cinta de clínker & 0,12 & 10 \\
Cámara II & 0,096 & 10 \\
\hline
\end{tabular}

que informa F. J. Mardulier, (32), y que se consiguieron con HEA-2, figuran en la tabla 4. Estos resultados demuestran la importancia de la forma de aplicar los aditivos de molienda, debiéndose realizar, en todo caso, ensayos en las fábricas.

\section{LOS EFECTOS DE LOS ADITIVOS DE MOLIENDA}

\subsection{Las bases}

H. M. v. Seebach, (36), ha descrito detalladamente los efectos de los aditivos de molienda durante la molienda de clínker. Ha comprobado que la reducción de las fuerzas de adhesión es la causa del mejor aprovechamiento de energía y que en las condiciones que predominan en un molino de bolas no se disminuye la resistencia a la molienda.

Los aditivos de molienda reducen el recubrimiento de las bolas y placas del molino, la formación de partículas secundarias del polvo finísimo y la adhesión de las partículas 
más finas a las gruesas. Así se mejora el efecto de trituración de los elementos molturadores y la separación de los granos suficientemente finos del material a moler. La reducción de las fuerzas adhesivas en el material no solamente tiene efectos durante el propio proceso de molienda, sino también durante el transporte, el desempolvado, el ensilado, el envasado, el embarque y el transporte al exterior.

\subsection{La influencia sobre el proceso de molienda}

\subsubsection{Tiempo de permanencia del material en el molino}

El tiempo de permanencia del material en un determinado molino tubular depende de las propiedades de flujo del material, de la resistencia de paso a través de los cuerpos molturadores, de los tabiques de separación y de la ventilación del molino. La velocidad de paso del material aumenta cuando éste se muele con más facilidad o cuando la resistencia de paso a través de los cuerpos molturados es más reducida. La relación de masas entre los cuerpos de molienda y el material a moler puede variar. El mayor aprovechamiento de energía se alcanza cuando esta relación es de $9: 1$, (33).

El tiempo medio de permanencia del material en el tubo del molino se averigua marcando con fluoresceína, (42). Se adiciona fluoresceína sódica en sọlución acuosa al material a la entrada del molino y se sacan muestras del flujo del material a la salida del molino. La concentración relativa de fluoresceína se mide por medio de un fotómetro.

Como tiempo medio de permanencia $t_{\mathrm{v}}$ del material en el tubo se da aquel que corresponde a la muestra con la mayor concentración de fluoresceína. El tiempo de permanencia así definido solamente coincide exactamente con el tiempo de permanencia medio estático cuando el espectro del tiempo de permanencia está repartido normalmente. Eso corresponde con suficiente exactitud en los molinos en circuito abierto, (43). En los ensayos realizados en molinos en circuito cerrado se obtuvieron los espectros de tiempo de permanencia que se representan en la figura 6. De ellos se desprende que la influencia de la fluoresceína reducida por los gruesos del retorno no modifica decisivamente la distribución de la concentración en la zona del primer máximo.

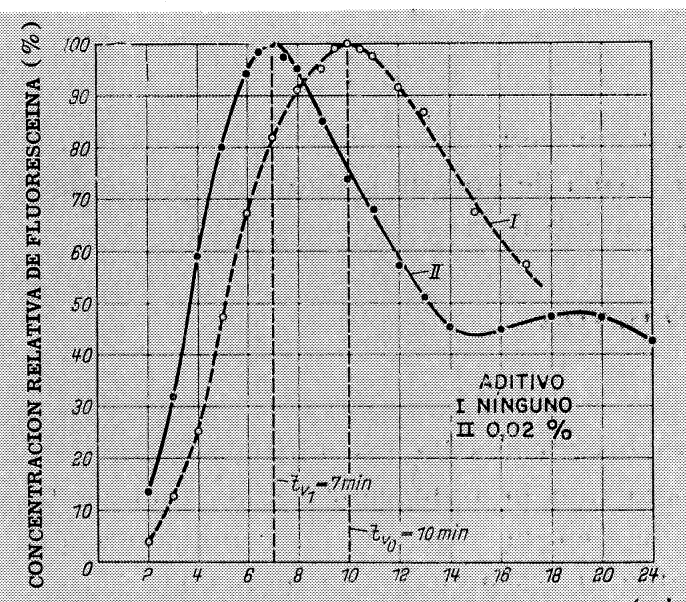

TOMA DE MUESTRAS CON LA ADICION DE FLUORESCEINA (min)

FIg. 6.-Medila del tlempo medilo de permanencla del material con $y$ sin aditivo en un mollino de cemento de $3,4 \times 1,25 \mathrm{~m}$.

\begin{tabular}{|c|c|c|c|c|}
\hline Estimación del trabajo & & & $\begin{array}{l}\sin \\
\text { aditivo }\end{array}$ & $\begin{array}{l}\text { eon } \\
\text { adilivo }\end{array}$ \\
\hline Masa de cuerpos de molienda & $\mathrm{M}_{\mathrm{K}}$ & t: & 125 & 125 \\
\hline Producción & D & $\mathrm{t} / \mathrm{h}$ & 48 & 55 \\
\hline Cantidad rechazada & a & $\mathrm{t} / \mathrm{h}$ & 72 & 71 \\
\hline Cantidad recirculada & U & $\mathrm{t} / \mathrm{h}$ & 120 & 126 \\
\hline Relación de recirculación & u & - & 2,5 & 2,3 \\
\hline Tiempo de retención & $t_{\mathrm{v}}$ & $\min$ & 10 & 7 \\
\hline Material dentro del mollino & $\mathrm{M}_{\mathrm{R}}$ & t & 20 & 14,7 \\
\hline Relación $\mathrm{M}_{\mathrm{K}} / \mathrm{M}_{\mathrm{R}}$ & & - & $6,3: 1$ & $8.5: 1$ \\
\hline
\end{tabular}


La masa del material $\mathrm{M}_{\mathrm{R}}$ en el tubo del molino se cálcula según la relación:

$$
\begin{array}{l|l}
M_{R}=\frac{U}{U} \cdot t_{v} & \begin{array}{l}
U=\text { carga circulante }(t / h) \\
t_{v}=\text { tiempo de permanencia }(\min ) ;
\end{array}
\end{array}
$$

y la relación exigida de los cuerpos de molienda al material a moler, según los datos de servicio indicados, es de 6,3:1, curva (I), para la molienda sin aditivos y de $8,5: 1$, curva (II), en el caso de emplear adiciones. Reducido el tiempo de permanencia por los aditivos de molienda de 10 a 7 minutos se crea un cambio en la relación de masas en el margen comprendido entre 8:1 y 10:1. El segundo ascenso de la curva II, que empieza a los 15 minutos, es producido por la fluoresceína que contiene el flujo de alimentación. El segundo máximo de la curva II se produce después de, aproximadamente, el doble del tiempo de permanencia $t_{v}$, más el tiempo necesario para el transporte de la carga circulante.

Un aumento considerable de la relación de masas por encima del valor 10:1 puede evitarse, por ejemplo, con un aumento de la carga circulante, con un llenado con cuerpos de molienda más finos o por reducción de la sección transversal abierta de las rejillas de paso y de descarga. Una relación de masas demasiado grande o demasiado pequeña lleva a un rendimiento insatisfactorio.

\subsubsection{Trabajo en el separador}

Como consecuencia de la reducción de las fuerzas adhesivas entre partículas, el separador trabaja mejor y, con ello, menos material fino ya molido se devuelve de nuevo al molino.

La figura 7 representa las curvas de trabajo de un separador centrífugo ZUB 25 durante la molienda sin aditivo de molienda, (I), y con aditivo de molienda, (II). Se fabricó un cemento con una superficie específica de unos $2.400 \mathrm{~cm}^{2} / \mathrm{g}$. En la marcha de las curvas de separación se reconoce una mejora. En este ensayo la diferencia no es muy grande, porque el separador, debido a la reducida carga de 34 a $38 \mathrm{t} / \mathrm{h}$, separa bastante bien en ambos casos.

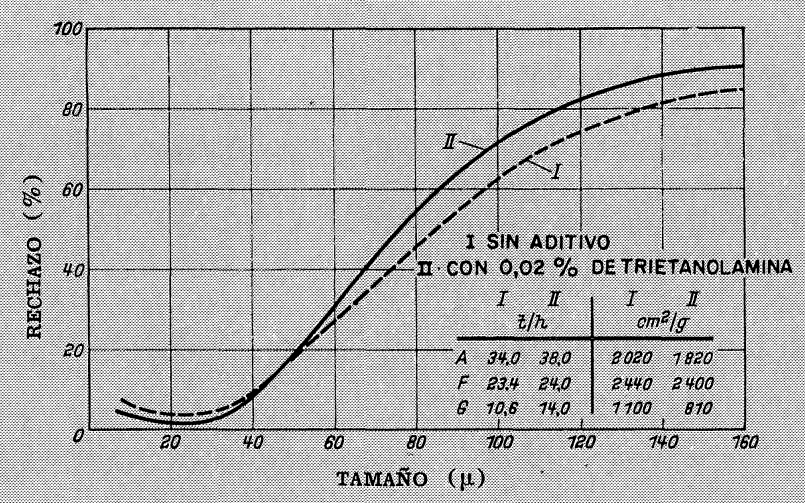

Fig. 7.-Curva granulométrica de un separador centrifugo.

La mejora se ve más claramente en un cemento de alto horno 275 con unos $3.500 \mathrm{~cm}^{2} / \mathrm{g}$ (fig. 8). Sin aditivo, curva (I), un $60 \%$ del material alimentado salió del separador sin se- 


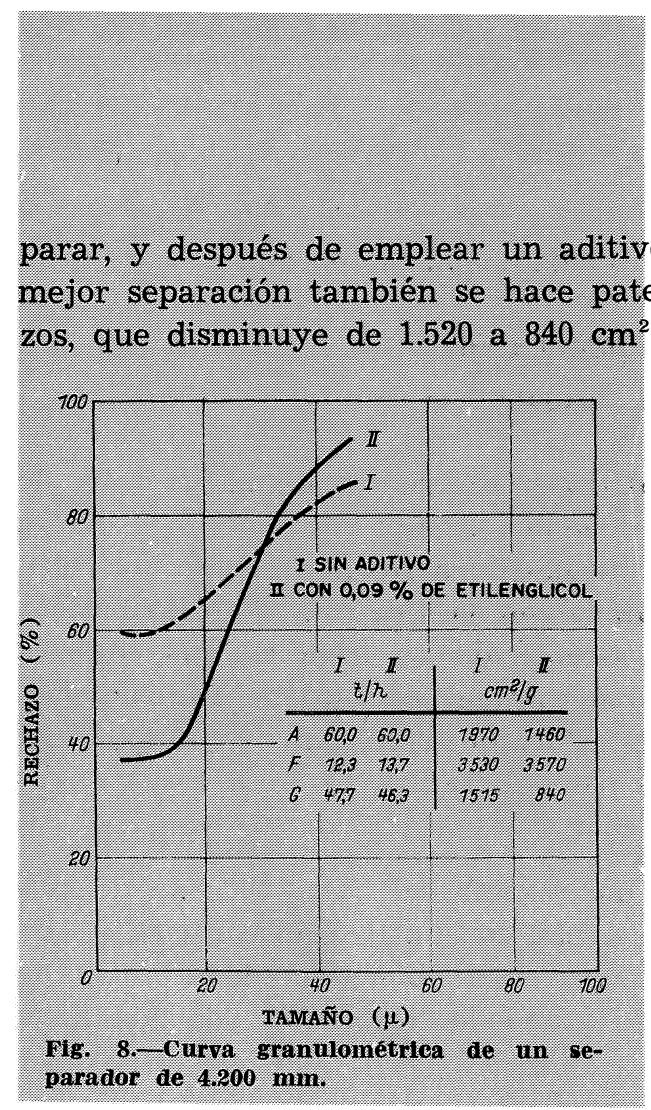

La mejor separación tiene efectos favorables para el proceso de molienda, sobre todo cuando el separador trabaja con carga elevada. Pero no siempre tendrá como consecuencia un aumento del rendimiento del molino. El tubo del molino sólo puede realizar una trituración determinada, y el aprovechamiento óptimo de energía con la finura exigida en el material acabado obliga a una determinada distribución granulométrica del material de alimentación que sólo puede obtenerse si el separador crea un rechazo determinado en cantidad y distribución granulométrica. Esa exigencia no tiene que depender necesariamente de una separación idealmente exacta.

\subsection{Influencia sobre el proceso después de la molienda}

La fluidez más elevada del cemento facilita la elevación neumática. y la salida del silo. También acelera la carga de vehículos que, más tarde, se descargan con más rápidez y completamente. Por esto, algunas fábricas emplean aditivos de molienda sólo para mejorar la fluidez.

A veces, las mangas de tejido de los filtros, en general bastante impenetrables, no pueden retener las partículas más finas de polvo. Esto puede remediarse empleando mangas de más baja permeabilidad al aire. También, al envasar el cemento en sacos pueden producirse dificultades porque el cemento no libera rápidamente el aire incorporado, llenándose los sacos sin haber alcanzado su peso reglamentario. En este caso, el proceso de llenado de la máquina tiene que hacerse más lento.

Puede comprobarse la variación en la fluideż al utilizar aditivos de molienda por médıo de un canal neumático de ensayo, (45), en que se mide el recorrido de una muestra de cemento con diferente velocidad de aire en el tejido del canal.

Mientras que este ensayo caracteriza el comportamiento del cemento durante el transporte neumático, el aparato en la figura 9 caracteriza cualitativamente las fuerzas adhesivas que determinan el flujo desde el estado de reposo. En un matraz de cristal con vibración se compacta una muestra de cemento ; luego, el matraz se fija en un dispositivo giratorio. El número de revoluciones necesario para desmoronar el cemento apelmazado se llama índice Pack-Set y es una medida de las propiedades de fluidez del cemento.

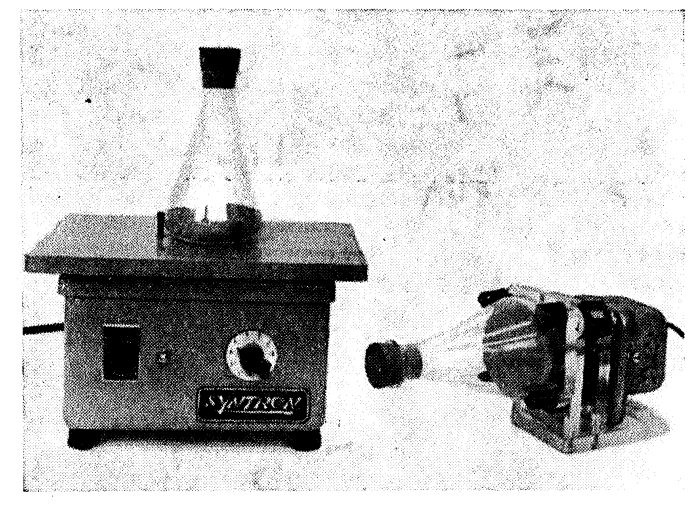

Fig. 9.-Aparato "Pack-Set" para conocer el comportamiento al flujo del cemento. 


\subsection{Modificación de las propiedades del cemento}

Las condiciones de molturación modificadas por los aditivos de molienda tienen sus efectos sobre la distribución granulométrica del cemento y, por lo tanto, pueden influir en el endurecimiento.

Con la misma superficie específica, en general, se reduce el residuo en los tamices de 0,09 y $0,06 \mathrm{~mm}$, de modo que no es posible prever el desarrollo de resistencias con los valores de los residuos sobre dichos tamices al pasar a la molienda con aditivos.

El cambio de la distribución granulométrica en el campo de tamaños muy finos no es muy grande en general, y con frecuencia no se puede comprobar con seguridad. Las distribuciones granulométricas de cementos con la misma superficie específica están representadas, como ejemplo, en la figura 10. En la mayoría de los casos se observan resistencias iniciales más elevadas, más no muy importantes; pero hasta ahora no se ha podido comprobar si eso se debe a la distinta granulometría o a las reacciones entre los aditivos de molienda y el clínker.

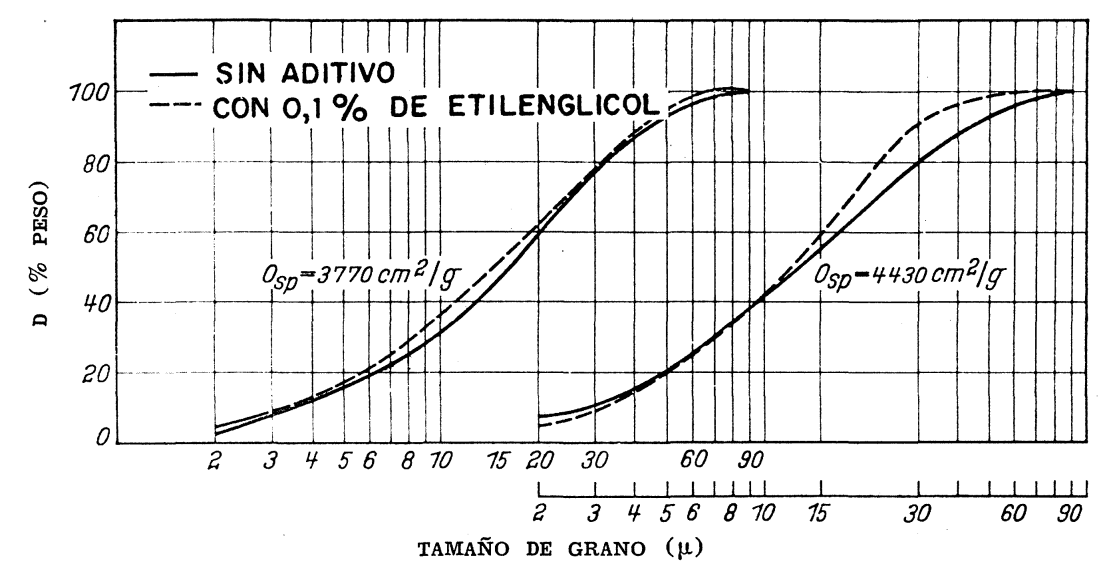

Fig. 10.-Curva granulométrica acumulada de los cementos.

\section{ENSAYO DE LOS ADITIVOS DE MOLIENDA}

Según los ensayos realizados por H. M. v. Seebach, (36), las medidas de absorción junto con mediciones de fuerza adhesiva, constituyen la mejor previsión de la eficacia de un aditivo.

Para el ensayo de las influencias en la calidad del cemento son aconsejables moliendas en un molino de laboratorio con calefacción. Debería exigirse del suministrador el ensayo prescrito en la norma DIN 1164 , (46), para aditivos, en lo que se refiere al contenido de cloruros y a la comprobación de que estos productos son inocuos respecto a la corrosión del acero. Este ensayo, por ejemplo, lo realiza la Bundesanstalt für Materialprüfung (BAM) en Berlín.

\section{UTILIDAD DE LOS ADITIVOS DE MOLIENDA}

Al juzgar la economía en el empleo de coadyuvantes de molienda en general, únicamente se tienen en cuenta los costos de energía. Pero es más correcto tomar en consideración 
el costo total del proceso. El aumento de rendimiento necesario $\Delta \mathrm{D}$, en $\%$, para compensar los costos, se cálcula según la expresión:

$$
\Delta \mathrm{D}=\frac{100}{\left(\mathrm{~K}_{\mathrm{M}} / \mathrm{K}_{\mathrm{MH}}\right)-1}, \%
$$

o

$$
\Delta \mathrm{D}=\frac{100 \cdot \mathrm{K}_{\mathrm{MH}}}{\mathrm{K}_{\mathrm{M}}-\mathrm{K}_{\mathrm{MH}}}, \% \text {; }
$$

donde:

$\mathrm{K}_{\mathrm{M}} \quad$ son los costos de la corriente eléctrica (en $\mathrm{DM} / \mathrm{t}$ ) calculados según el trabajo específico (en $\mathrm{kWh} / \mathrm{t}$ ) y el precio de la corriente (en $\mathrm{DM} / \mathrm{kWh}$ ); o los costos totales de molienda, incluyendo los costos de desgaste, personal, materiales de servicio, mantenimiento de instalaciones, amortización e intereses.

$\mathrm{K}_{\mathrm{MH}}$ representa el costo de los aditivos de molienda (en $\mathrm{DM} / \mathrm{t}$ ) que resulta de la cantidad de aditivo (en $\mathrm{kg} / \mathrm{t}$ ), o sea, en \%, y del precio de los aditivos (en $\mathrm{DM} / \mathrm{kg}$ ) que hoy se encuentra entre 0,80 y $1,40 \mathrm{DM} / \mathrm{kg}$.

El importe de ahorro $\mathrm{E}$ (en DM/t), función del aumento del rendimiento (en \%), resulta de la ecuación:

$$
\mathrm{E}=\mathrm{K}_{\mathrm{M}} \cdot \frac{100}{100+\Delta \mathrm{D}}-\mathrm{K}_{\mathrm{MH}}, \quad(\mathrm{DM} / \mathrm{t})
$$

y se ve fácilmente en la figura 11. En los casos I y II se ha supuesto un aumento del rendimiento de un $18 \%$. Si los costos de corriente son de $3,20 \mathrm{DM} / \mathrm{t}$ y los costos de aditivos de $0,65 \mathrm{DM} / \mathrm{t}$, entonces los costos de molienda aumentan en $0,16 \mathrm{DM} / \mathrm{t}$. Con los mismos valores iniciales de $\Delta \mathrm{D} \mathrm{y}_{\mathrm{MH}}$, y para unos costos de molienda de 7,80 DM/t, los costos de molienda disminuyen por $0,47 \mathrm{DM} / \mathrm{t}$. Del diagrama también puede desprenderse, sin dificultad, el aumento del rendimiento necesario para la compensación de costos.

Los cálculos mencionados solamente tienen validez si se basan en el mismo tiempo de marcha del molino y si puede aprovecharse la máxima capacidad de producción.

En cambio, un aumento del rendimiento tiene también ventajas económicas cuando por un tiempo de marcha más corto del molino pueden evitarse puntas de corriente y conseguirse tarifas más adecuadas. Los costos de personal disminuyen cuando pueden evitarse horas extraordinarias.

Es decir, debido a las diferentes condiciones previas en las distintas fábricas, la decisión de emplear aditivos de molienda puede depender también de factores que no se tienen en cuenta en las ecuaciones [2] y [3], o sea, en la figura 11.

\section{RESUMEN}

Desde hace unos 40 años aproximadamente, se emplean aditivos de molienda líquidos para mejorar la producción de los molinos tubulares y su rendimiento energético durante la molienda de los cementos. En 1968, alrededor del $10 \%$ del cemento producido en la República Federal se preparó con aditivos de molienda, principalmente glicoles y aminas. Estos líquidos, que cuestan de 0,80 a $1,40 \mathrm{DM} / \mathrm{kg}$, se añaden en forma no diluida o en so- 
lución acuosa a razón de menos de $1 \mathrm{~kg} / \mathrm{t}(1 \%)$. Los mejores resultados se han obtenido por una pulverización en la cámara de molienda fina. La mejora de la producción que se logra es de 10 a $30 \%$ en la molienda del portland 375 y del 25 al $50 \%$ en la del portland 475. La molienda de los cementos de alto horno sólo se mejora ligeramente.

La acción de los aditivos de molienda se basa en una reducción de las fuerzas de adherencia. No disminuye la resistencia a la molienda del clínker.

El material a moler se mueve más fácilmente. Los separadores realizan un trabajo mucho más limpio. El manejo neumático del cemento es mucho más fácil; los silos y los vehículos-cisterna pueden vaciarse rápida y completamente. Las mejoras en la producción del molino rebajan los costes de la molienda.

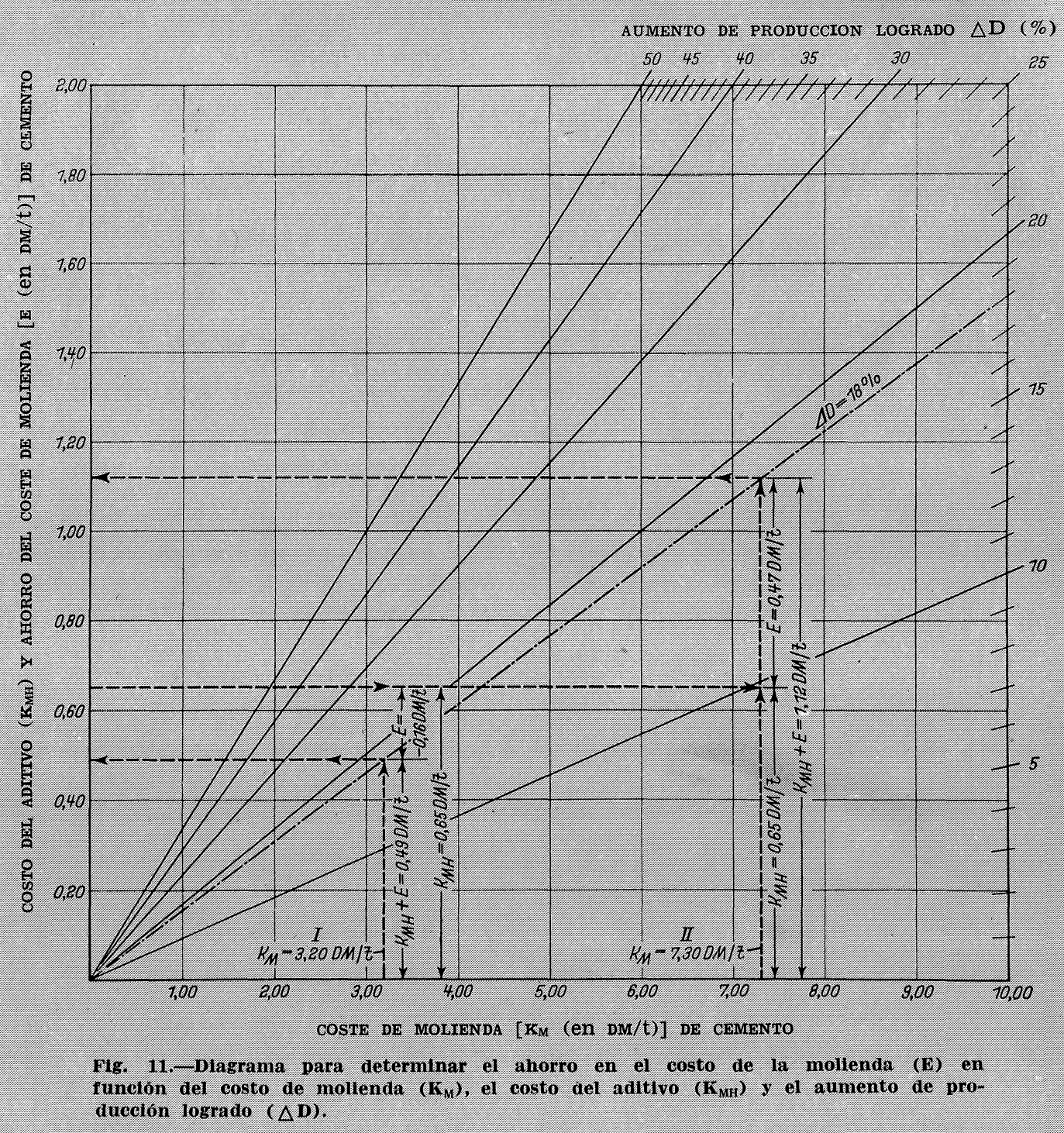




\section{BIBLIOGRAFIA}

(1) Brit. Pat. Nr. 350538 vom 12. März 1930. Erf.: J. F. Goddard. Improvements relating to process for grinding materials.

(2) US. Pat. Nr. 2141571 vom 9. November 1935. Erf.: H. L. Kennedy und J. G. Mark. Grinding of cement clinker.

(3) DP. Nr. 924965 vom 19. Jull 1952. Erf.: K. Trautmann. Verfahren zum Fe:nmahlen von Zementen ... unter Verwendung von als Mahlhilfen wirkenden Stoffen.

(4) Brit. Pat. Nr. 788155 vom 21. April 1954. Erf.: J. L. v. Eichborn. Improvements in the mechanical treatment of materials.

(5) Osterr. Pat. Nr. 190022 vom 15. Juli 1956. Erf.: J. L. v. Eich born. Chemisches Verfahren zur Verbesserung von Werkstoffgefüge verändernden oder auflösenden mechanischen Vorgängen in Gasatmosphäre.

(6) DPA Nr. 1158348 vom 21. April 1960. Erf.: G. R. Fagerholt. Verfahren zum Vermahlen kristalliner Stoffe.

(7) US. Pat. Nr. 3068110 vom 11. Dezember 1962. Erf.: G. R. Fagerholt. Method of grinding portland cement using a phenolic compound as a grinding aid.

(8) DPA Nr. 1220775 vom 21. Januar 1965. Erf.: F. G. Serafin und V. H. Dodson. Zusatzstoff für Portlandzement.

(9) Canad. Pat. Nr. 778814 vom 4. Mai 1967. Erf.: V. H. Dodson und F, G. Serafin. Cement additives composed of ethanolamine salts.

(10) K e n n e d y, H. I.: Portland cement-effects of catalysis and dispersion. Ind. Engng. Chem. 28 (1936), 963-969.

(11) D a w le y, E. R.: Effect of TDA on the rate of grinding portland cement. Pit and Quarry, Jull 1939, 44-46 und 63.

(12) $\mathrm{H}$ a e g e rma n n, G.: Zusatzmittel zur Verbesserung der Verarbeitbarkeit von Beton. Zement 32 (1943), 125-140.

(13) B o n d, F. C., und A g the, F. T.: Deleterious coatings of the media in dry ball milling. Mining Technol. 4 (1960).

(14) S w e i t z e r, C. W., und C raig, A. E.: Colloidal carbon as a grinding aid in portland cement manufacture. Ind. Engng. Chem. 32 (1940), 751-756.

(15) D a w l e y, E. R.: Tests on cement clinker alds. Cement Lime Manufact. 17 (1944), 1-4.

(16) M a s e l, A.: Ein neuer Zusatz zur Verbesserung der Mahlleistung. Baustoff-Ind., Moskau, 67 (1953), 3.

(17) T a g g a r t, A. F.: Handbook of mineral dressing. John Wiley \& Sons, Inc., New York 1948, Section 3 A: Cement.

(18) B I a n k s, R. F., und Kennedy, H. L.: The technology of cement and concrete. John Wiley \& Sons, Inc., New York 1955, Ch. 6: Additions and admixtures.

(19) Rose, H. E., und S ullivan, R. M. E.: A treatise on the internal mechanics of ball, tube and rod mills. Constable
\& Co. L.td., London 1957, Ch. 9: The role of additives in milling.

(20) Chemical Engineer's Handbook. McGraw-Hill Book Company, Inc., New York, Toronto, London 1950, Crushing and grinding.

(21) G rzy mek, J., Gus t a w, K., und O s t a p, K.: Der Einfluß oberflächenwirksamer Zusatzstoffe auf den Mahlvorgan fluß oberflachenwin Gips 52 (1965), 1-7.

(22) G h i g i, G., und R a b o t $t$ in o, L.: Experiments on cement clinker grinding with additives. 2. Europ. Symp. „Zerkleinern“, Amsterdam 1966, Bericht C 2.
(23) K u d r ja wze wa, N. L., und $\mathrm{Ch}$ o d a kow, G. Ss.: Einfluß oberflächenaktiver Substanzen auf das Mahlen. von Klinker. Doklady Akad. Nauk SSSR 156 (1964), 437-440; ref. in: Chemisches Zentralblatt 137 (1966), S. 1974, Nr. 2197.

(24) Vé n u a t, M.: Note sur les agents de mouture. Rev. Matér. Construct. 1967, 622-623; CERILH Publicat. Technique Nr. 184.

(25) $\mathrm{S} \mathrm{h}$ a w, K.: Use surface-active agents for rapid fine grinding of cement clinkers. Cement, Lime Gravel 40 (1965), 358-359.

(26) Mospan, J. G.: Die Suche nach wirksamen chemischen Zusătzen. Zement (russ.) 30 (1964) H. 4, 5-6.

(27) Rojak, S. M., P P rozki, W., und Mazuew, N.: Verbesserung der Zementmahlung durch Zusatz oberflăchenaktiver Stoffe. Zement (russ.) 30 (1964) H. 5, 5-8.

(28) P i rovarov, V. J., und A ki mov, v. A.: Intensivierung des Klinkermahlvorganges mit Hilfe von oberflächenaktiven Stoffen. Zement (russ.) 31 (1965) H. 5, 18-19.

(29) S i d o ट̌ en k o, J. M., $Z$ a v g or od nif. N. S., und B u din I. $K$.: Intensivierung des Mahlvorgangs fur Klinker. Zement (russ.) 34 (1968) H. 5, 3-4

(30) Bojarsin ov, E. G., und Guse va, G. M.: Wirksames Klinkermahlen in Muhlen mit Umlauf. Zement (russ.) 33 (1967) H. 4, 16-17.

(31) Mardulie r, F. J.: The mechanism of grinding aids. Proceedings ASTM 61 (1961), 1078--1093.

(32) M a rdulie r, F. J.: Make grinding aids work for you. Rock Products, MaI 1967.

(33) M a r d u l i e r, F. J.: Balance-key to mill/separator operation. Rock Products 4th annual industry operations seminar Chikago, Ill., 1. - 4. 12. 1968

(34) z i e g l e r, E.: Beeinflussung der Mahlbarkeit von Festkörpern durch Zusatz von oberflächenaktiven Stoffen. Schriftenreihe der Zementindustrie, Heft 19 (1956). Verein Deutscher Zementwerke e. V., Düsseldorf.

(35) G ö t t e, A., und $\mathrm{Z}$ i e g l e r, E.: Versuche zur Herabsetzung des Zerkleinerungswiderstandes fester Stoffe durch gasförmige und dampfförmige Zusatzmittel. VDI-Zeitschrift 98 (1956), 373-376.

(36) v. S e e b a c h, H. M.: Die Wirkung von Dämpfen organischer Flüssigkeiten bel der Zerkleinerung von Zementklinker in Kugelmuhlen. Zement-Kalk-Gips 22 (1969), 202-211.

(37) Verein Deutscher Zementwerke e. V., Forschungsinstitut der Zementindustrie, Düsseldorf, Tätigkeitsbericht 1962, 61-64.

(38) dgl. Tätigkeitsbericht 1963/64, 80-81.

(39) dgl. Tätigkeitsbericht 1965/66, 33-34.

40) $\mathrm{Z}$ eise 1, H. G.: Entwicklung eines Verfahrens zur Bestimmung der Mahlbarkeit. Schriftenreihe der Zementindustrie, Heft 14 (1953), S. 31-72. Verein Deutscher Zementwerke e. V., Dusseldorf.

(41) L u dw $1 \mathrm{~g}$, N. C., und O'H a ra, L. M.: How to get better additives. Rock Products 64 (1961) H. 7, 102-108.

42) $\mathrm{C}$ h on e, B.: Mesure du temps de rétention dans le broyeur. Rev. Matér. Construct. Aug./Sept. 1968, S. 330-331.

(43) P a u l s e n, H.: Leistungsaufnahme und Axialdispersion des Mahlgutes in der Ein-Kammer-Kugelmunle. Dissertation TH Karlsruhe 1969.

44) S chönert, K.: Uber die mathematische Behandlung des Mühlen-Sichter-Kreislaufs. Interne Sitzung des Arbeitsausschusses "Zerkleinerungstechnik" der Verfahrenstechnischen Gesellschaft im VDI am 14. April 1969 in Leverkusen.

(45) F i s c h e r, W.: Fließverhaiten von Zement. Zement-Kalk Gips 20 (1967), 138-139.

46) DIN 1164: Portland-, Eisenportland-, Hochofen-, Trabzement, Blatt 1, Entwurf Juil 1967, Abschnitt 2.2.6, Fußnote 4 